\title{
Bayesian network marker selection via the thresholded graph Laplacian Gaussian prior
}

\author{
Qingpo Cai* ${ }^{*}$ Jian Kang ${ }^{\dagger}$ and Tianwei $\mathrm{Yu}^{\ddagger}$
}

\section{Appendix}

This supplementary material contains the proofs for Theorems 3.1 and 3.2 in the manuscript.

\subsection{Regularity conditions}

We start with notation. Let $p_{n}$ be the number of predictors that grow with $n$ and $q$ be the number of confounding variables. Let $\epsilon_{n} \in(0,1]$ be a sequence of positive numbers such that $n \epsilon_{n}^{2} \rightarrow \infty$. Denote $d_{0}\left(f, f^{*}\right)=\int f^{*} \log \left(f^{*} / f\right)$ as the Kullback-Leibler divergence between two densities $f$ and $f^{*}$ and define $d_{t}\left(f, f^{*}\right)=t^{-1}\left(\int f^{*}\left(f^{*} / f\right)^{t}-1\right)$ for any $t>0$. It is straightforwar to see that $d_{t}$ decreases to $d_{0}$ as $t$ decreases to 0 . Define $\hat{\pi}(\epsilon)=\pi\left[d\left(p, p^{*}\right) \geq \epsilon \mid D_{n}\right]$, for any $\epsilon>0$. We define a pre-specified upper bound $\bar{r}_{n}$ for

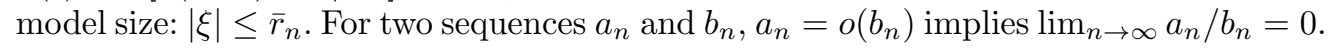
Denote by $a \vee b$ the max number between $a$ and $b$. Denote by $r_{n}$ the model size of $\xi_{n}$ $\left(\left|\xi_{n}\right|=r_{n}\right)$. Let $\mathcal{P}_{n}=\left\{f\left(y ; \xi, \beta_{\xi}, \boldsymbol{\omega}\right):|\xi| \leq \bar{r}_{n},\left|\beta_{j}\right|_{j \in \xi} \leq C_{n},\left|\omega_{k}\right|_{k \in Q} \leq C_{n}\right\}$, for some $C_{n}>0$. Denote by $N\left(\epsilon_{n}, \mathcal{P}_{n}\right)$ the minimal number of Hellinger balls of radius $\epsilon_{n}$ that are needed to cover $\mathcal{P}_{n}$. Define $\triangle\left(r_{n}\right)=\inf _{\xi:|\xi|=r_{n}} \sum_{j: j \notin \xi}\left|\beta_{j}^{*}\right|$ and $D(R)=$ $1+R \times \sup _{|h| \leq R}\left|a^{\prime}(h)\right| \times \sup _{|h| \leq R}\left|g^{-1}(h)\right| . \sigma_{\alpha}^{2}$ and $\sigma_{\omega}^{2}$ are variance terms defined in the prior. $L$ is the graph Laplacian matrix and $\varepsilon$ is a positive number. $\pi$ is a probability measure. $p$ and $p^{*}$ are two density functions.

We consider the following conditions:

(C1) $\bar{r}_{n} \log \left(1 / \epsilon_{n}^{2}\right)=o\left(n \epsilon_{n}^{2}\right)$;

(C2) $\bar{r}_{n} \log p_{n}=o\left(n \epsilon_{n}^{2}\right)$;

(C3) $r_{n}=o\left(p_{n}\right)$;

(C4) $\triangle\left(r_{n}\right)=o\left(\epsilon_{n}^{2}\right)$;

(C5) $\left(\bar{r}_{n}+q\right) \log D\left(\left(\bar{r}_{n}+q\right)\left(n \epsilon_{n}^{2}\left(\sigma_{\alpha}^{2} \vee \sigma_{\omega}^{2}\right) / 2\right)^{1 / 2}\right)=o\left(n \epsilon_{n}^{2}\right)$;

(C6) $1 \leq r_{n} \leq \bar{r}_{n}<p_{n}$;

\footnotetext{
${ }^{*}$ Department of Biostatistics and Bioinformatics, Emory University, Atlanta, GA 30322, USA

${ }^{\dagger}$ Department of Biostatistics, University of Michigan, Ann Arbor, MI 48109, USA jiankang@umich.edu

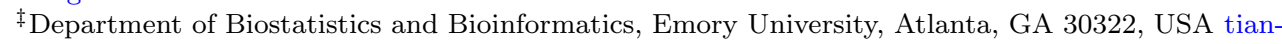
wei.yu@emory.edu
} 
(C7) Let $\Lambda_{\gamma}=\left(\mathbf{L}+\varepsilon \mathbf{I}_{p}\right)^{-1}$. After a permutation for rows and columns, $\Lambda_{\gamma}$ can be decomposed as

$$
\tilde{\Lambda}_{\gamma}=\left(\begin{array}{ll}
\tilde{\Lambda}_{11} & \tilde{\Lambda}_{12} \\
\tilde{\Lambda}_{21} & \tilde{\Lambda}_{22}
\end{array}\right)
$$

where $\tilde{\Lambda}_{11}$ is a scalar; $\tilde{\Lambda}_{21}$ is of dimension $\left(p_{n}-1\right) \times 1$ and $\tilde{\Lambda}_{22}$ is of dimension $\left(p_{n}-1\right) \times\left(p_{n}-1\right)$. Set $\sigma_{1}^{2}=\tilde{\Lambda}_{\gamma}^{R}=\tilde{\Lambda}_{11}-\tilde{\Lambda}_{12} \tilde{\Lambda}_{22}^{-1} \tilde{\Lambda}_{21}$. Consider the same procedure for $\tilde{\Lambda}_{22}$ and we obtain $\sigma_{2}^{2}=\tilde{\Lambda}_{22}^{R}$. Repeat the above procedure, then a sequence $\left\{\sigma_{1}^{2}, \sigma_{2}^{2}, \cdots, \sigma_{r_{n}}^{2}\right\}$ can be obtained. We assume that $\inf \left\{\sigma_{j}^{2}\right\}_{j=1}^{r_{n}}>0$;

(C8) The upper bound of the covering number of $\mathcal{P}_{n}: \log \left\{\mathrm{N}\left(\epsilon_{n} / 4, \mathcal{P}_{n}\right)\right\} \leq n \epsilon_{n}^{2} / 16$;

(C9) $\pi\left(\mathcal{P}_{n}^{c}\right) \leq e^{-n \epsilon_{n}^{2} / 8}$

(C10) $\pi\left[p: d_{t}\left(p, p^{*}\right) \leq \epsilon_{n}^{2} / 64\right] \geq e^{-n \epsilon_{n}^{2} / 64}$ for some $t>0$.

Condition $(\mathrm{C} 7)$ is not a strong condition and we conduct an empirical study to show the correctness of condition $(\mathrm{C} 7)$. We generate scale free network and random network with different edge probability using $\mathrm{R}$ package igraph. The number of nodes ranges from 500 to 5,000 . We set $r_{n}=\sqrt{p_{n}}$ and fix $\varepsilon=10^{-5}$. Figure 1 shows the value of $\inf \left\{\sigma_{j}^{2}\right\}_{j=1}^{r_{n}}>0$ defined in condition (C7) with different number of nodes. As we can see, all infimum values are bounded away from 0 .

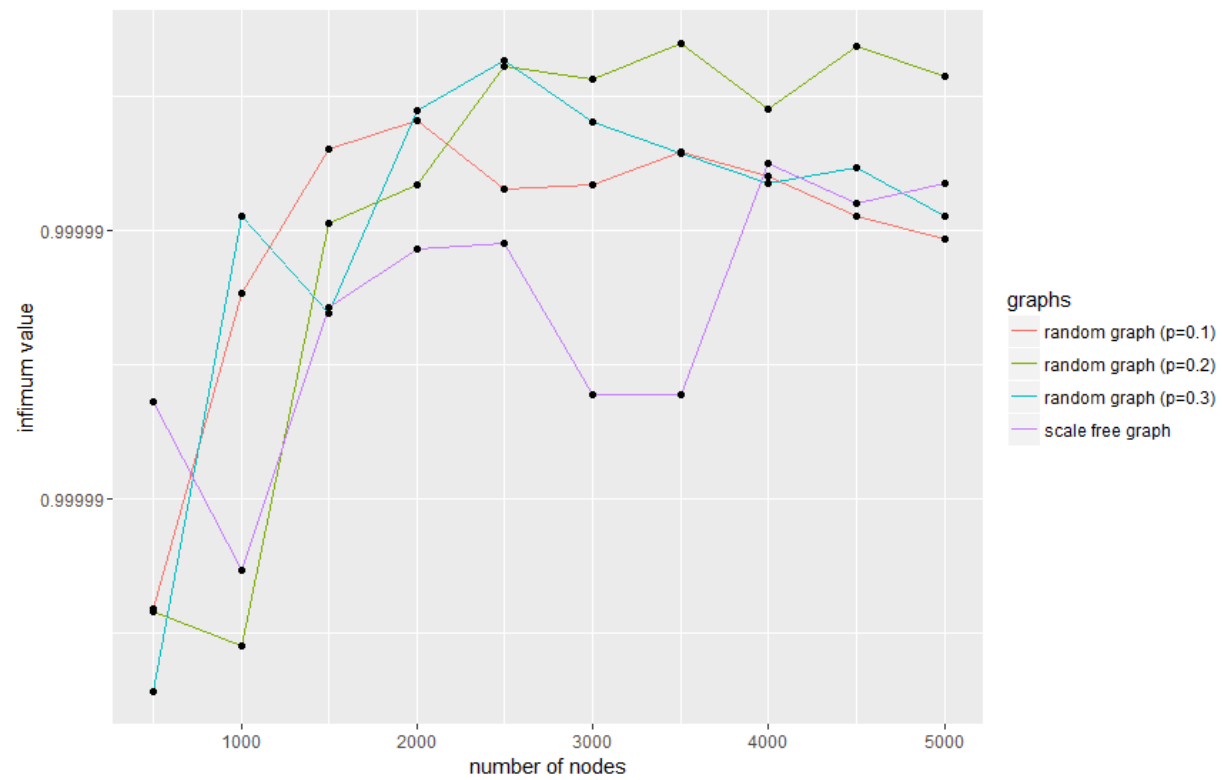

Figure 1: An illustration example for condition (C7) 
Given Theorem 3.1, the proof of Theorem 3.2 directly follows proof of Theorem 4 in Jiang (2007) and Theorem 1 in Song and Liang (2015). To prove Thoerem 3.1, we need to first introduce the following two lemmas.

Lemma 1. For a one dimension Gaussian random $Y \sim \mathrm{N}\left(0, \sigma^{2}\right)$ with $\sigma^{2}>0$, denote $P_{\sigma^{2}}(\mathcal{C})=P(Y \in \mathcal{C})$ for set $\mathcal{C} \in \mathcal{R}$. We have (i) $P_{\sigma^{2}}(\mathcal{C}) \leq P_{\sigma^{2}}(\mathcal{C}-z)$ for $\mathcal{C}=\{Y|| Y \mid>$ $\lambda\}$ and $z \in \mathcal{R}$; (ii) $\sigma_{1}^{2} \leq \sigma_{2}^{2} \Rightarrow P_{\sigma_{1}^{2}}(\mathcal{C}) \leq P_{\sigma_{2}^{2}}(\mathcal{C})$.

Proof. (i) First, without loss of generality, we assume $z>0$. Let $\phi(y)$ denote the density for $Y \sim \mathrm{N}\left(0, \sigma^{2}\right)$. Then we have

$$
\begin{aligned}
\mathrm{P}_{\sigma^{2}}(\mathcal{C})-\mathrm{P}_{\sigma^{2}}(\mathcal{C}-z) & =\int_{\lambda}^{\infty} \phi(y) d y+\int_{-\infty}^{-\lambda} \phi(y) d y-\int_{\lambda-z}^{\infty} \phi(y) d y-\int_{-\infty}^{-\lambda-z} \phi(y) d y \\
& =\int_{-\lambda-z}^{-\lambda} \phi(y) d y-\int_{\lambda-z}^{\lambda} \phi(y) d y=\int_{\lambda}^{\lambda+z} \phi(y) d y-\int_{\lambda-z}^{\lambda} \phi(y) d y \leq 0 .
\end{aligned}
$$

The inequality holds since $\phi(y)$ is symmetric around 0 and $\phi(y)$ is smaller in $[\lambda, \lambda+z]$ than $[\lambda-z, \lambda]$ since $\lambda>0$.

(ii) Assume $Y_{1} \sim \mathrm{N}\left(0, \sigma_{1}^{2}\right)$ and $Y_{2} \sim \mathrm{N}\left(0, \sigma_{2}^{2}\right)$. We can have $Y_{2} \stackrel{d}{=} Y_{1}+Z$ with $Z \sim \mathrm{N}\left(0, \sigma_{2}^{2}-\sigma_{1}^{2}\right)$ and $Y_{1}, Z$ are independent.

$$
\mathrm{P}_{\sigma_{2}^{2}}(\mathcal{C})=\mathrm{E}\left\{P_{\sigma_{1}^{2}}\left[Y_{1} \in \mathcal{C}-Z \mid Z\right]\right\} \geq \mathrm{E}\left\{P_{\sigma_{1}^{2}}\left[Y_{1} \in \mathcal{C}\right]\right\}=P_{\sigma_{1}^{2}}(\mathcal{C})
$$

Lemma 2. Suppose a p-dimensional multivariate Gaussian variable $Y \sim \mathrm{N}\left(\mathbf{0}_{p}, \Sigma\right)$. We partition $Y$ as

$$
\left(\begin{array}{l}
Y_{1} \\
Y_{2}
\end{array}\right) \sim\left(\begin{array}{ll}
\sigma_{11} & \sigma_{12} \\
\sigma_{21} & \Sigma_{22}
\end{array}\right)
$$

where $Y_{1}$ is of dimension one and $Y_{2}$ is of dimension $p-1 . \sigma_{11}, \sigma_{12}, \sigma_{21}$ and $\Sigma_{22}$ are the corresponding value, vector and sub-matrix from $\Sigma$. For $\mathcal{C}_{1}=\{y|| y \mid>\lambda\}$ and $\mathcal{C}_{2} \in \mathcal{R}^{p-1}$, We have:

$$
\mathrm{P}\left(Y_{1} \in \mathcal{C}_{1}, Y_{2} \in \mathcal{C}_{2}\right) \geq \mathrm{P}_{\sigma_{11.2}}\left(\mathcal{C}_{1}\right) \mathrm{P}_{\Sigma_{22}}\left(\mathcal{C}_{2}\right)
$$

where $\sigma_{11.2}=\sigma_{11}-\sigma_{12} \Sigma_{22}^{-1} \sigma_{21}$.

Proof. Note $V=Y_{1}-\sigma_{12} \Sigma_{22}^{-1} Y_{2} \sim \mathrm{N}\left(0, \sigma_{11.2}\right)$ and $Y_{2} \sim \mathrm{N}\left(0, \Sigma_{22}\right) . V, Y_{2}$ are independent.

$$
\begin{aligned}
\mathrm{P}\left(Y_{1} \in \mathcal{C}_{1}, Y_{2} \in \mathcal{C}_{2}\right) & =\mathrm{E}\left\{\mathrm{P}\left[V \in \mathcal{C}_{1}-\sigma_{12} \Sigma_{22}^{-1} Y_{2}, Y_{2} \in \mathcal{C}_{2} \mid Y_{2}\right]\right\} \\
& =\mathrm{E}\left\{\mathrm{P}\left[V \in \mathcal{C}_{1}-\sigma_{12} \Sigma_{22}^{-1} Y_{2} \mid Y_{2}\right] I_{\mathcal{C}_{2}}\left(Y_{2}\right)\right\} \\
& \geq \mathrm{E}\left\{\mathrm{P}\left[V \in \mathcal{C}_{1} \mid Y_{2}\right] I_{\mathcal{C}_{2}}\left(Y_{2}\right)\right\}=\mathrm{P}_{\sigma_{11.2}}\left(\mathcal{C}_{1}\right) \mathrm{P}_{\Sigma_{22}}\left(\mathcal{C}_{2}\right)
\end{aligned}
$$




\subsection{Proof for Theorem 3.1}

Proof. We have $\gamma \sim \mathrm{N}\left(0, \sigma_{\gamma}^{2} \Lambda_{\gamma}\right)$. Set $\gamma_{\xi_{n}}=\left\{\gamma_{j}, j \in \xi_{n}\right\}$ as a vector of length $\left|\xi_{n}\right|$ and $\gamma_{-\xi_{n}}=\left\{\gamma_{j}, j \notin \xi_{n}\right\}$. Write the thresholding parameter as $\lambda_{n}$ to denote that $\lambda_{n}$ might increase as $n$ increases. Let $\mathcal{C}=\mathcal{C}_{1} \cup \mathcal{C}_{2}=\left\{\bigcup_{j \in \xi_{n}}\left\{\left|\gamma_{j}\right|>\lambda_{n}\right\}\right\} \bigcup\left\{\bigcup_{j \notin \xi_{n}}\left\{\left|\gamma_{j}\right| \leq \lambda_{n}\right\}\right\}$.

$$
\pi\left(\xi=\xi_{n}\right)=\pi\left(\gamma_{\xi_{n}} \in \mathcal{C}_{1}, \gamma_{-\xi_{n}} \in \mathcal{C}_{2}\right)
$$

Denote $\xi_{n}=\left(i_{1}, \cdots, i_{\left|\xi_{n}\right|}\right)$. Set $\mathcal{C}_{i_{1}}=\left\{\left|\gamma_{i_{1}}\right|>\lambda_{n}\right\}, \mathcal{C}_{-i_{1}}=\mathcal{C} \backslash \mathcal{C}_{1}$. Let $\sigma_{i_{1}}=\sigma_{11}-$ $\sigma_{12} \Sigma_{22}^{-1} \sigma_{21}$ for

$$
\left(\begin{array}{c}
\gamma_{i_{1}} \\
\gamma_{-i_{1}}
\end{array}\right) \sim\left(\begin{array}{cc}
\sigma_{11} & \sigma_{12} \\
\sigma_{21} & \Sigma_{22}
\end{array}\right)
$$

According to Lemma 2, we have :

$$
\begin{aligned}
\pi\left(\xi=\xi_{n}\right) & =\pi\left(\gamma_{\xi_{n}} \in \mathcal{C}_{1}, \gamma_{-\xi_{n}} \in \mathcal{C}_{2}\right)=\pi\left(\gamma_{i_{1}} \in \mathcal{C}_{i_{1}}, \gamma_{-i_{1}} \in \mathcal{C}_{-i_{1}}\right) \\
& \geq \pi_{\sigma_{i_{1}}}\left(\gamma_{i_{1}} \in \mathcal{C}_{i_{1}}\right) \pi_{\Sigma_{22}}\left(\gamma_{-i_{1}} \in \mathcal{C}_{-i_{1}}\right)
\end{aligned}
$$

Similarly, we can apply the same procedure to $\pi_{\Sigma_{22}}\left(\gamma_{-i_{1}} \in \mathcal{C}_{-i_{1}}\right)$ until we have gone through all the elements in $\xi_{n}$. Then we have

$$
\pi\left(\xi=\xi_{n}\right) \geq\left\{\Pi_{j \in \xi_{n}} \pi_{\sigma_{j}}\left(\left|\gamma_{j}\right|>\lambda_{n}\right)\right\} \times \pi_{\Sigma_{-\xi_{n}}}\left(\gamma_{-\xi_{n}} \in \mathcal{C}_{2}\right),
$$

where $\Sigma_{-\xi_{n}}=\sigma_{\gamma}^{2} \Lambda_{\gamma}(i, j)_{i, j \notin \xi_{n}}$. By (C7), we have $\sigma_{1}^{2}=\inf _{j \in \xi_{n}} \sigma_{j}>0$. According to Lemma $1, \pi_{\sigma_{j}}\left(\left|\gamma_{j}\right|>\lambda_{n}\right) \geq \pi_{\sigma_{1}^{2}}\left(\left|\gamma_{j}\right|>\lambda_{n}\right)$, for all $j \in \xi_{n}$.

According to Anderson (1955), for a $p$-dimensional multivariate Gaussian random variable $Y \sim \mathrm{N}_{p}(0, \Sigma)$, we have $\Sigma_{1} \leq \Sigma_{2} \Rightarrow P_{\Sigma_{1}}(\mathcal{C}) \geq P_{\Sigma_{2}}(\mathcal{C})$ for every centrally symmetric convex set $\mathcal{C}$. In our case, $\mathcal{C}_{2}$ is a centrally symmetric convex set. According to Chung (1997), the eigenvalue for a $p$ dimension Graph Laplacian matrix $L$ is : $0=$ $\lambda_{1} \leq \lambda_{2} \leq \cdots \leq \lambda_{p}$. So the maximum eigenvalue for $\Sigma_{-\xi_{n}}$ is smaller than $\sigma_{\gamma}^{2} / \epsilon$ since the maximum eigenvalue for $\Lambda_{\gamma}$ is smaller than $1 / \epsilon$. Then we could have $\pi_{\Sigma_{-\xi_{n}}}\left(\gamma_{-\xi_{n}} \in\right.$ $\left.\mathcal{C}_{2}\right) \geq \pi_{\sigma_{\gamma}^{2} / \epsilon I_{p_{n}-\left|\xi_{n}\right|}}\left(\gamma_{-\xi_{n}} \in \mathcal{C}_{2}\right)=\left(\pi\left(|Y| \leq \lambda_{n}\right)\right)^{p_{n}-\left|\xi_{n}\right|}$ where $Y \sim \mathrm{N}\left(0, \sigma_{\gamma}^{2} / \epsilon\right)$. Note that $\pi\left(\xi=\xi_{n}\right) \geq\left(\pi_{\sigma_{1}^{2}}\left(\left|\gamma_{j}\right|>\lambda_{n}\right)\right)^{\left|\xi_{n}\right|}\left(\pi_{\sigma_{\gamma}^{2} / \epsilon}\left(\left|\gamma_{j}\right| \leq \lambda_{n}\right)\right)^{p_{n}-\left|\xi_{n}\right|}$. Take $\lambda_{n}$ such that $\min \left\{\pi_{\sigma_{1}^{2}}\left(\left|\gamma_{j}\right|>\lambda_{n}\right), \pi_{\sigma_{\gamma}^{2} / \epsilon}\left(\left|\gamma_{j}\right|>\lambda_{n}\right)\right\}=\left|\xi_{n}\right| / p_{n}$. Then we have $-\log \pi\left(\xi=\xi_{n}\right) \leq$ $-\left|\xi_{n}\right| \log \left(\left|\xi_{n}\right| / p_{n}\right)-\left(p_{n}-\left|\xi_{n}\right|\right) \log \left(1-\left|\xi_{n}\right| / p_{n}\right) \leq\left|\xi_{n}\right| \log p_{n}+\left|\xi_{n}\right|=o\left(n \epsilon_{n}^{2}\right)$, since $\left|\xi_{n}\right|=o\left(p_{n}\right)$ and $\left|\xi_{n}\right| \log p_{n} \leq \bar{r}_{n} \log p_{n}=o\left(n \epsilon_{n}^{2}\right)$.

Given $\xi_{n}$ and $\sigma_{\alpha}^{2}$, we have $\alpha_{j} \stackrel{\text { i.i.d }}{\sim} \mathrm{N}\left(0, \sigma_{\alpha}^{2}\right)$ and $\beta_{j}=\alpha_{j}$ for $j \in \xi_{n}$. For $j \in \xi_{n}$, denote $\phi$ the infimum of the density for $\mathrm{N}\left(0, \sigma_{\alpha}^{2}\right)$ for all $\left\{\beta_{j}^{*} \pm \eta \epsilon_{n}^{2} /\left|\xi_{n}\right|\right\}_{j \in \xi_{n}}$. So $-\log \pi\left(\boldsymbol{\beta}_{\xi} \in\right.$ $\left.B\left(\xi_{n}, \eta\right) \mid \xi=\xi_{n}\right) \leq-\left|\xi_{n}\right| \log \left(2 \phi \eta \epsilon_{n}^{2} /\left|\xi_{n}\right|\right) \leq\left|\xi_{n}\right| \log \left|\xi_{n}\right|+C\left|\xi_{n}\right|+\left|\xi_{n}\right| \log \left(1 / \epsilon_{n}^{2}\right)=o\left(n \epsilon_{n}^{2}\right)$ where $C$ is some constant, since $\left|\xi_{n}\right| \log \left(1 / \epsilon_{n}^{2}\right) \leq \bar{r}_{n} \log \left(1 / \epsilon_{n}^{2}\right)=o\left(n \epsilon_{n}^{2}\right)$ and $\left|\xi_{n}\right| \log \left|\xi_{n}\right| \leq$ $\bar{r}_{n} \log p_{n}=o\left(n \epsilon_{n}^{2}\right)$.

By Mill's ratio, for all $j \in \xi_{n}$, we have $\pi\left(\left|\beta_{j}\right|>C_{n}\right) \leq 2 e^{-C_{n}^{2} /\left(2 \sigma_{\alpha}^{2}\right)} / \sqrt{2 \pi C_{n}^{2} / \sigma_{\alpha}^{2}}$ with $\beta_{j} \sim \mathrm{N}\left(0, \sigma_{\alpha}^{2}\right)$. Choose $C_{n}=\sqrt{n \epsilon_{n}^{2} \sigma_{\alpha}^{2} / 2}$. Then we have $\pi\left(\left|\beta_{j}\right|>C_{n}\right) \leq e^{-n \epsilon_{n}^{2} / 4}$ for a large enough $n$. This completes the proof for Theorem 3.1. 


\subsection{Proof for Thoerem 3.2}

Next, we show the proof for Theorem 3.2.

Following from Theorem 6 of Jiang (2005) and Proposition 1 of Jiang (2007), we have the Following Lemma 3.

Lemma 3 Assume there is a sequence $\epsilon_{n} \in(0,1]$ such that $n \epsilon_{n}^{2} \rightarrow \infty$. If for all large enough $n$, conditions $(\mathrm{C} 8)-(\mathrm{C} 10)$ hold:

Then we have:

(i) $P\left\{\hat{\pi}\left(\epsilon_{n}\right) \geq 2 e^{-n \epsilon_{n}^{2} \min \{1 / 32, t / 64\}}\right\} \leq 2 e^{-n \epsilon_{n}^{2} \min \{1 / 32, t / 64\}}$;

(ii) $E \hat{\pi}\left(\epsilon_{n}\right) \leq 4 e^{-n \epsilon_{n}^{2} \min \{1 / 16, t / 32\}}$

Next, we prove Theorem $\mathbf{3 . 2}$ by verifying that our model settings satisfy conditions (C8), (C9), (C10).

\section{Proof. Verfiy condition (C10):}

Consider the case for $t=1$. The GLM density is $f(y, h)=\exp \{a(h) y+b(h)+c(y)\}$. Let $p^{*}=f\left(y, h^{*}\right)$ with $h^{*}=\mathbf{x}^{T} \boldsymbol{\beta}^{*}+\mathbf{z}^{T} \boldsymbol{\omega}^{*}$ and $p_{\xi_{n}}=f\left(y, h_{\xi_{n}}\right)$ with $h_{\xi_{n}}=\mathbf{z}^{T} \boldsymbol{\omega}+$ $\mathbf{x}_{\xi_{n}}^{T} \boldsymbol{\beta}_{\xi_{n}}=\mathbf{z}^{T} \boldsymbol{\omega}+\sum_{j \in \xi_{n}} x_{j} \beta_{j}$. As shown in the proof of Theorem 4 of Jiang (2007), when $h^{*}$ and $h_{\xi_{n}}$ are close enough, $d_{1}\left(p_{\xi_{n}}, p^{*}\right)$ can be put in the form as $d_{1}\left(p_{\xi_{n}}, p^{*}\right)=$ $E_{x} g(\tilde{h})\left(h^{*}-h_{\xi_{n}}\right)$, where $g$ is a continuous derivative function in a neighborhood of $h^{*}$ and $\tilde{h}$ is an intermediate point between $h^{*}$ and $h_{\xi_{n}}$.

Note that $r_{n}$ is the model size for $\xi_{n}\left(\left|\xi_{n}\right|=r_{n}\right)$ and $Q=\{1, \cdots, q\}$. We have $\left|\tilde{h}-h^{*}\right| \leq\left|h^{*}-h_{\xi_{n}}\right| \leq\left|\sum_{k \in Q} z_{k}\left(\omega_{k}-\omega_{k}^{*}\right)\right|+\left|\sum_{j \in \xi_{n}} x_{j}\left(\beta_{j}-\beta_{j}^{*}\right)\right|+\left|\sum_{j \notin \xi_{n}} x_{j} \beta_{j}^{*}\right| \leq$ $q M \max _{k \in Q}\left\{\left|\omega_{k}-\omega_{k}^{*}\right|\right\}+r_{n} M \max _{j \in \xi_{n}}\left\{\left|\beta_{j}-\beta_{j}^{*}\right|\right\}+M \Delta\left(r_{n}\right)$. If there exists small enough $\delta_{1}$ and $\delta_{2}$ such that $\beta_{j} \in\left(\beta_{j}^{*} \pm \delta_{1}\right)$ for all $j \in \xi_{n}$ and $\omega_{k} \in\left(\omega_{k}^{*} \pm \delta_{2}\right)$ for all $k \in Q$, we could have that $\left|\tilde{h}-h^{*}\right| \leq\left|h^{*}-h_{\xi_{n}}\right| \leq M \Delta\left(r_{n}\right)+M r_{n} \delta_{1}+M q \delta_{2}$. Here we have $\Delta\left(r_{n}\right)=o\left(\epsilon_{n}^{2}\right)$ by condition $(\mathrm{C} 4)$.

For sufficiently small $\delta_{1}, \delta_{2},|g(\tilde{h})|$ is bounded since $|\tilde{h}| \leq\left|h^{*}\right|+\left|\tilde{h}-h^{*}\right| \leq B_{0}+$ $M \Delta\left(r_{n}\right)+M r_{n} \delta_{1}+M q \delta_{2}$ is bounded, where $B_{0}=\lim _{n \rightarrow \infty} \sum_{j=1}^{p_{n}}\left|\beta_{j}^{*}\right|$. Then we could have $d_{1}\left(p_{\xi_{n}}, p^{*}\right) \leq C\left(M \Delta\left(r_{n}\right)+M r_{n} \delta_{1}+M q \delta_{2}\right)$ for some constant $C$ and small enough $\delta_{1}, \delta_{2}$. Take $\delta_{1}=\eta_{1} \epsilon_{n}^{2} /\left(M r_{n}\right)$ and $\delta_{2}=\eta_{2} \epsilon_{n}^{2} /(M q)$ for small enough $\eta_{1}, \eta_{2}$. Then we could have $d_{1}\left(p_{\xi_{n}}, p^{*}\right) \leq \epsilon_{n}^{2} / 64$ for large enough $n$ and small enough $\eta_{1}, \eta_{2}$. So we can conclude that $\left\{\xi=\xi_{n}, \beta_{j} \in\left(\beta_{j}^{*} \pm \eta_{1} \epsilon_{n}^{2} /\left(M r_{n}\right)\right), j \in \xi_{n}, \omega_{k} \in\left(\omega_{k}^{*} \pm \eta_{2} \epsilon_{n}^{2} /(M q)\right), k \in\right.$ $Q\} \subset\left\{p: d_{1}\left(p_{\xi_{n}}, p^{*}\right) \leq \epsilon_{n}^{2} / 64\right\}$.

As shown in the proof of Theorem 1, we have $-\log \pi\left(\xi=\xi_{n}\right)=o\left(n \epsilon_{n}^{2}\right)$ and $-\log \pi\left(\beta_{\xi} \in\left\{\beta_{j}^{*} \pm \eta_{1} \epsilon_{n}^{2} /\left(M\left|\xi_{n}\right|\right)\right\}_{j \in \xi_{n}} \mid \xi=\xi_{n}\right)=o\left(n \epsilon_{n}^{2}\right)$. Similarly, it's easy to show $-\log \pi\left(\boldsymbol{\omega} \in\left\{\omega_{k}^{*} \pm \eta_{2} \epsilon_{n}^{2} /(M q)\right\}_{k \in Q}\right)=o\left(n \epsilon_{n}^{2}\right)$. Then we can have $-\log \pi\left\{p: d_{1}\left(p_{\xi_{n}}, p^{*}\right) \leq\right.$ $\left.\epsilon_{n}^{2} / 64\right\}=o\left(n \epsilon_{n}^{2}\right)$.

Verify condition (C8): For each model $\xi$ in $\mathcal{P}_{n}$, there are $|\xi|+q$ nonzero elements, with each element bounded by $\left[-C_{n}, C_{n}\right]$. It takes at most $\left[C_{n} / \delta+1\right]^{|\xi|+q}$ balls with 
radius $\delta(\delta>0)$ to cover the parameter space of model $\xi$. For each model $\xi$ with size $|\xi|=r$, there are at most $p_{n}^{r}$ models for $r=0,1, \cdots, \bar{r}_{n}$. Then we could have that the number of radius- $\delta$ balls $N(\delta)$ needed to cover the parameter space in $\mathcal{P}_{n}$ is at most $\sum_{r=0}^{\bar{r}_{n}} p_{n}^{r}\left[C_{n} / \delta+1\right]^{r+q}$, which is bounded by $\left(\bar{r}_{n}+1\right) p_{n}^{\bar{r}_{n}}\left[C_{n} / \delta+1\right]^{\bar{r}_{n}+q}$. This means that for any density in $\mathcal{P}_{n}$ that can be represented by a set of regression parameters $\left\{\omega_{k}^{u}\right\}_{1}^{q},\left\{u_{j}\right\}_{1}^{p_{n}}$, it must fall in these $N(\delta)$ balls with center $\left\{\omega_{k}^{v}\right\}_{k=1}^{q},\left\{v_{j}\right\}_{j=1}^{p_{n}}$, i.e. $\left\{\omega_{k}^{v} \pm\right.$ $\delta\}_{k=1}^{q},\left\{v_{j} \pm \delta\right\}_{j=1}^{p_{n}}$, where $u_{j}$ and $v_{j}$ are zero for the same model $\xi$ and $|\xi| \leq \bar{r}_{n}$.

Consider the corresponding GLM densities $f_{u}=\exp \left\{a\left(h_{u}\right) y+b\left(h_{u}\right)+c(y)\right\}$ and $f_{v}=$ $\exp \left\{a\left(h_{v}\right) y+b\left(h_{v}\right)+c(y)\right\}$ with $h_{u}=\sum_{k=1}^{q} z_{k} \omega_{k}^{u}+\sum_{j=1}^{p_{n}} x_{j} u_{j}$ and $h_{v}=\sum_{k=1}^{q} z_{k} \omega_{k}^{v}+$ $\sum_{j=1}^{p_{n}} x_{j} v_{j}$. It is straightforward to show that the Hellinger distance between $f_{u}$ and $f_{v}$ is smaller than the square root KL divergence, $d\left(f_{u}, f_{v}\right) \leq \sqrt{d_{0}\left(f_{u}, f_{v}\right)}$. The KL divergence is $d_{0}\left(f_{u}, f_{v}\right)=E_{\mathbf{z}, \mathbf{x}} \int f_{v}\left(\log f_{v}-\log f_{u}\right) \nu_{y}(d y)$. Integrate out $y$ and apply a Taylor expansion. We can show that $d_{0}\left(f_{u}, f_{v}\right) \leq E_{\mathbf{z}, \mathbf{x}}\left(a^{\prime}(\tilde{h}) \psi\left(h_{v}\right)+b^{\prime}(\tilde{h})\right)\left(h_{v}-h_{u}\right)$, where $\psi=-b^{\prime} / a$ and $\tilde{h}$ is an intermediate point between $h_{v}$ and $h_{u}$. By definition, we could have $h_{u}, h_{v}, \tilde{h}$ are all bounded by $\left(\bar{r}_{n}+q\right) C_{n}$. Note that $\left|h_{u}-h_{v}\right|=\mid \sum_{k \in Q} z_{k}\left(\omega_{k}^{u}-\right.$ $\left.\omega_{k}^{v}\right)|+| \sum_{j \in \xi} x_{j}\left(u_{j}-v_{j}\right) \mid \leq M\left(\bar{r}_{n}+q\right) \delta$ since $\left|u_{j}-v_{j}\right| \leq \delta$ and $\left|\omega_{k}^{u}-\omega_{k}^{v}\right| \leq \delta$. Therefore,

$$
d_{0}\left(f_{u}, f_{v}\right) \leq 2 \sup _{|h| \leq\left(\bar{r}_{n}+q\right) C_{n}}\left|a^{\prime}(h)\right| \times \sup _{|h| \leq\left(\bar{r}_{n}+q\right) C_{n}}|\psi(h)| M\left(\bar{r}_{n}+q\right) \delta
$$

and

$$
d\left(f_{u}, f_{v}\right) \leq\left\{2 \sup _{|h| \leq\left(\bar{r}_{n}+q\right) C_{n}}\left|a^{\prime}(h)\right| \times \sup _{|h| \leq\left(\bar{r}_{n}+q\right) C_{n}}|\psi(h)| M\left(\bar{r}_{n}+q\right) \delta\right\}^{1 / 2}
$$

Let $\delta=\epsilon_{n}^{2} /\left\{32 \sup _{|h| \leq\left(\bar{r}_{n}+q\right) C_{n}}\left|a^{\prime}(h)\right| \times \sup _{|h| \leq\left(\bar{r}_{n}+q\right) C_{n}}|\psi(h)| M\left(\bar{r}_{n}+q\right)\right\}$ and we have $d\left(f_{u}, f_{v}\right) \leq \epsilon_{n} / 4$. So we have the Hotelling covering number:

$$
\begin{aligned}
\log N\left(\epsilon_{n} / 4, \mathcal{P}_{n}\right) & \leq \log N(\delta) \\
& \leq \log \left(\bar{r}_{n}+1\right)+\bar{r}_{n} \log p_{n}+\bar{r}_{n} \log \left(32 \epsilon_{n}^{-2} \sup _{|h| \leq \bar{r}_{n} C_{n}}\left|a^{\prime}(h)\right| \times \sup _{|h| \leq \bar{r}_{n} C_{n}}|\psi(h)|+1\right) \\
& \leq \log \left(\bar{r}_{n}+1\right)+\bar{r}_{n} \log p_{n}+\left(\bar{r}_{n}+q\right) \log D\left(\left(\bar{r}_{n}+q\right) C_{n}\right)+\bar{r}_{n} \log 32 .
\end{aligned}
$$

Since $\bar{r}_{n} \log p_{n}=o\left(n \epsilon_{n}^{2}\right)$ and $\left(\bar{r}_{n}+q\right) \log D\left(\left(\bar{r}_{n}+q\right) C_{n}\right)=o\left(n \epsilon_{n}^{2}\right)$ with $C_{n}=\sqrt{n \epsilon_{n}^{2}\left(\sigma_{\alpha}^{2} \vee \sigma_{\omega}^{2}\right) / 2}$, we have $\log N\left(\epsilon_{n} / 4, \mathcal{P}_{n}\right)=o\left(n \epsilon_{n}^{2}\right)$.

Verify condition (C9): $\mathcal{P}_{n}=\left\{f\left(y ; \xi, \beta_{\xi}\right):|\xi| \leq \bar{r}_{n},\left|\beta_{j}\right|_{j \in \xi} \leq C_{n},\left|\omega_{k}\right|_{k \in Q} \leq C_{n}\right\}$, for some $C_{n}>0$. By the definition of $\bar{r}_{n}$, we have $\pi\left(|\xi|>\bar{r}_{n}\right)=0$. So we could get $\pi\left(\mathcal{P}_{n}^{c}\right) \leq \max _{\xi:|\xi| \leq \bar{r}_{n}} \pi(\xi) \pi\left(\cup_{j \in \xi}\left[\left|\beta_{j}\right|>C_{n}\right] \mid \xi\right)+\pi\left(\cup_{k \in Q}\left[\left|\omega_{k}\right|>C_{n}\right]\right)$. By Mill's ratio, we have $\pi\left(\left|\beta_{j}\right|>C_{n}\right) \leq 2 e^{-C_{n}^{2} /\left(2 \sigma_{\alpha}^{2}\right)} / \sqrt{2 \pi C_{n}^{2} / \sigma_{\alpha}^{2}}$ for $\beta_{j} \sim N\left(0, \sigma_{\alpha}^{2}\right)$ and $j \in \xi$. Similarly, $\pi\left(\left|\omega_{k}\right|>C_{n}\right) \leq 2 e^{-C_{n}^{2} /\left(2 \sigma_{\omega}^{2}\right)} / \sqrt{2 \pi C_{n}^{2} / \sigma_{\omega}^{2}}$ for $\omega_{k} \sim N\left(0, \sigma_{\omega}^{2}\right)$ and $k \in Q$. Choose $C_{n}=$ $\sqrt{n \epsilon_{n}^{2}\left(\sigma_{\alpha}^{2} \vee \sigma_{\omega}^{2}\right) / 2}$. Then we have $\pi\left(\left|\beta_{j}\right|>C_{n}\right) \leq e^{-n \epsilon_{n}^{2} / 4}$ and $\pi\left(\left|\omega_{k}\right|>C_{n}\right) \leq e^{-n \epsilon_{n}^{2} / 4}$ for large enough $n$. So $\pi\left(\mathcal{P}_{n}^{c}\right) \leq\left(2+\bar{r}_{n}+q\right) e^{-n \epsilon_{n}^{2} / 4} \leq e^{-n \epsilon_{n}^{2} / 8}$ for large enough $n$, since $\log \left(2+\bar{r}_{n}+q\right) \leq \bar{r}_{n} \log p_{n}=o\left(n \epsilon_{n}^{2}\right)$.

This completes the proof for Theorem 3.2. 


\section{Sensitivity Analysis}

We mainly performed two formal sensitivity analyses for simulated data: 1) fixed the prior of $\epsilon$ as log-normal $(-5,3)$ make mild changes in the inverse gamma hyperpriors for $\sigma_{\gamma}^{2}$ and $\sigma_{\alpha}^{2}$ to four settings: $\operatorname{IG}(0.01,0.01), \operatorname{IG}(0.005,0.005), \operatorname{IG}(0.001,0.001)$ and $\operatorname{IG}(0.1,0.1) ; 2)$ fixed the prior of $\sigma_{\gamma}^{2}$ and $\sigma_{\alpha}^{2}$ at $\operatorname{IG}(0.01,0.01)$ and make changes in the log-normal priors of $\epsilon$ to four settings: $\log$-normal $(-5,3), \log$-normal $(-5,4), \log$ normal $(-4,3)$ and log-normal $(-4,4)$. We compared the posterior mean and $95 \%$ CI of the effects of true informative nodes in the following tables. We concluded that the posterior distribution is not sensitive to mild changes of the prior specifications. We also conducted a similar sensitivity analysis for the analysis of TCGA data and drew the same conclusion.

\begin{tabular}{l|ll}
\multicolumn{3}{c}{ Fix $\epsilon \sim \log -\operatorname{normal}(-5,3)$} \\
& mean & $95 \% \mathrm{Cl}$ \\
\hline $\mathrm{IG}(0.01,0.01)$ & -2.28 & $(-4.13,-0.47)$ \\
$\mathrm{IG}(0.005,0.005)$ & -2.41 & $(-4.01,-0.74)$ \\
$\mathrm{IG}(0.001,0.001)$ & -2.23 & $(-3.74,-0.61)$ \\
IG(0.1,0.1) & -2.25 & $(-4.03,-0.47)$
\end{tabular}

\begin{tabular}{l|ll}
\multicolumn{2}{c}{ Fix $\sigma_{\gamma}^{2}, \sigma_{\alpha}^{2} \sim \operatorname{IG}(0.01,0.01)$} \\
& mean & $95 \% \mathrm{Cl}$ \\
\hline Log-normal $(-5,3)$ & -2.28 & $(-4.13,-0.47)$ \\
Log-normal $(-5,4)$ & -1.73 & $(-3.26,-0.24)$ \\
Log-normal $(-4,3)$ & -2.35 & $(-3.91,-0.81)$ \\
Log-normal $(-4,4)$ & -2.01 & $(-3.44,-0.60)$
\end{tabular}

\section{Posterior interval coverage}

We also conduct an analysis on posterior credible interval coverage for coefficients in simulation studies. For each of the 50 replicates in simulation studies, we randomly select 3 non-zero coefficients and 3 zero coefficients and calculate the coverage of the $95 \%$ credible interval for those 6 selected coefficients. See Table 1 for the final results. We conclude that the coverage is accurate for all simulation scenarios.

\section{References}

Anderson, T. W. (1955). "The integral of a symmetric unimodal function over a symmetric convex set and some probability inequalities." Proceedings of the American Mathematical Society, 6(2): 170-176.

Chung, F. R. (1997). Spectral graph theory, volume 92. American Mathematical Soc. 
Table 1: Average 95\% posterior credible interval coverage for randomly selected coefficients in simulation studies

\begin{tabular}{llll}
\hline & & Linear regression & Logistic regression \\
\hline$p=33$ & Type 1 & 0.970 & 0.883 \\
& Type 2 & 0.950 & 0.930 \\
\multirow{2}{*}{ scale-free } & Type 1 & 0.876 & 0.800 \\
& Type 2 & 0.890 & 0.807 \\
\hline & disconnected & 0.890 & 0.780 \\
& & 0.877 & 0.797
\end{tabular}

Jiang, W. (2005). "Bayesian variable selection for high dimensional generalized linear models." Technical report, Technical Report 05-02, Dept. Statistics, Northwestern Univ. Available at newton. stats. northwestern. edu/ jiang/tr/glmone2. tr. pdf.

- (2007). "Bayesian variable selection for high dimensional generalized linear models: convergence rates of the fitted densities." The Annals of Statistics, 35(4): 1487-1511.

Song, Q. and Liang, F. (2015). "A split-and-merge Bayesian variable selection approach for ultrahigh dimensional regression." Journal of the Royal Statistical Society: Series B (Statistical Methodology), 77(5): 947-972. 